\title{
INTEGRATED SHORELINE EXTRACTION APPROACH WITH USE OF RASAT MS AND SENTINEL-1A SAR IMAGES
}

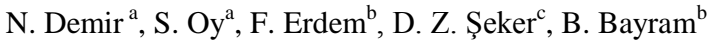 \\ a Akdeniz University, Space Science and Technologies, Antalya, Turkey- nusretdemir@akdeniz.edu.tr,selenoy171@gmail.com \\ b Yildiz Technical University, Dept. of Geomatic Engineering, Istanbul, Turkey, f5016010@ std.yildiz.edu.tr; bayram@yildiz.edu.tr \\ c Istanbul Technical University, Department of Geomatics Engineering, 34469, Maslak Istanbul Turkey, seker@itu.edu.tr
}

\section{Commission III, WG III/2}

KEYWORDS: SAR, Shoreline, Multispectral image, RASAT, Fuzzy clustering

\begin{abstract}
:
Shorelines are complex ecosystems and highly important socio-economic environments. They may change rapidly due to both natural and human-induced effects. Determination of movements along the shoreline and monitoring of the changes are essential for coastline management, modeling of sediment transportation and decision support systems. Remote sensing provides an opportunity to obtain rapid, up-to-date and reliable information for monitoring of shoreline. In this study, approximately $120 \mathrm{~km}$ of AntalyaKemer shoreline which is under the threat of erosion, deposition, increasing of inhabitants and urbanization and touristic hotels, has been selected as the study area. In the study, RASAT pansharpened and SENTINEL-1A SAR images have been used to implement proposed shoreline extraction methods. The main motivation of this study is to combine the land/water body segmentation results of both RASAT MS and SENTINEL-1A SAR images to improve the quality of the results. The initial land/water body segmentation has been obtained using RASAT image by means of Random Forest classification method. This result has been used as training data set to define fuzzy parameters for shoreline extraction from SENTINEL-1A SAR image. Obtained results have been compared with the manually digitized shoreline. The accuracy assessment has been performed by calculating perpendicular distances between reference data and extracted shoreline by proposed method. As a result, the mean difference has been calculated around 1 pixel.
\end{abstract}

\section{INTRODUCTION}

Shorelines are important features for land/water resources management, safe navigation, geographical mapping and coastal monitoring. Satellite imagery is the valuable data source for shoreline extraction, and there are freely available satellite images both from multispectral and radar sensors. Combined use of passive and active sensors would allow using advantages of a different wavelength. Synthetic Aperture Radar (SAR) images are a polar-orbiting satellite and operate in all-weather, day-and-night conditions with monitoring for land and water areas. Since the coastal areas have windy and cloudy weather conditions, SAR data is much advantageous compared to the multispectral imagery. On the other hand, processing of SAR images is challenging compared to the multispectral images, since they contain high level noise and speckles.

In recent years, there are many types of research were conducted related to coastline change detection using SAR images. Lee and Jurkevich (1990) proposed using an edge detection method supported by an edge-tracing algorithm to detect a coastline from low- resolution Synthetic Aperture Radar (SAR) images. Mason and Davenport (1996) proposed a semiautomatic method for detecting the shoreline from ERS-1 SAR images. This method integrated with an active contour model and the contrast ratio filter detector. Niedermeier et al. (2000) developed a detection algorithm based on wavelet and active contour methods. Yu and Acton (2004) delineated coastlines from space borne polarimetric SAR imagery of coastal urban areas using a diffusion based method. Kim et al. (2007) studied waterline detection in the intertidal areas using synthetic aperture radar (SAR) images and field measurements. Liu and Jezek (2004) improved an automated coastline extraction technique by merging Canny edge detection and locally adaptive thresholding methods. Shu et al. (2010) offered extraction shoreline from RADARSAT-2 imagery which was a semi-automated method. This method was combined morphological filters and segmentation. Xing et al. (2012) recommended a snake based method for an effective coastline extraction technique. To improve motion performance, it was developed a new internal and external energy function by them. Zhang et al. (2013) implemented an algorithm which is the combination of geometric active contour models and quadtree segmentation for extraction of coastline.

Paes et al. (2015) proposed a method for detect shoreline from RadarSAT-2 SAR data by using k-means clustering algorithm and Canny edge detection filter. Liu et al. (2016) improved a method for coastline extraction for wide-swath SAR imagery, which used the modified K-means method and an adaptive coarse-fine object-based region-merging (MKAORM). Modava and Akbarizadeh (2017) presented a novel coastline extraction procedure from SAR images utilizing spatial fuzzy clustering and the active contour method.

In this study, a new fuzzy approach is proposed to extract shorelines from SENTINEL-1A imagery. RASAT multispectral image is used for estimation of the parameters. The proposed approach is tested on the Antalya-Kemer shoreline.

\section{MATERIALS AND METHODS}

\subsection{Used Data and Study Area}

In the study, around $120 \mathrm{~km}$ of Antalya-Kemer shoreline located southern part of the Turkey, has been selected as the study area. The coast has the typical Mediterranean climate which is hot, dry weather and warm sea. The shore consists of 
different types of geological features mostly stony rather than sand. The selected study area is displayed in Figure 1.
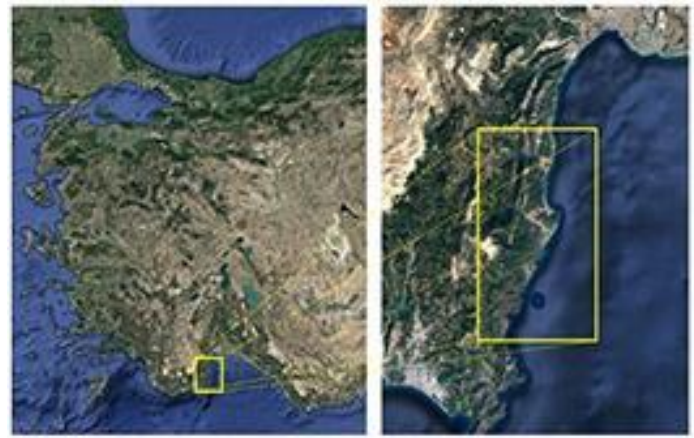

Figure 1.The study area

In the study, a RASAT pansharpened image and SENTINEL1 A SAR image were used as main data. Both images were acquired in August 2016. At the first step of the process, they have been co-registered to each other for the elimination of shifts in $\mathrm{X}$ and $\mathrm{Y}$. Random Forest classifier method has been applied on RASAT image to extract the shorelines, and the results are used to estimate the fuzzy parameters to extract the shorelines from SENTINEL-1A image. RASAT Earth Observation Satellite is the second remote sensing satellite of Turkey and designed by The Scientific and Technological Research Council of Turkey (TUBITAK), launched in August 2011. The technical specifications of RASAT satellite are listed in Table 1 (Erdogan et al. 2016).

Table 1. Technical specifications of RASAT satellite

\begin{tabular}{|l|l|}
\hline \multirow{2}{*}{ Spatial resolution } & Panchromatic- 7.5 m \\
\cline { 2 - 2 } & Multispectral - 15 m \\
\hline Spectral bands $(\mu \mathrm{m})$ & $0.42-0.73$ (Panchromatic) \\
\hline & 1st Band: 0.42-0.55 (Blue) \\
\hline & 2nd Band: 0.55-0.58 (Green) \\
\hline & 3rd Band: 0.58-0.73 (Red) \\
\hline Radiometric resolution & 8 bits \\
\hline Revisit time & 4 days \\
\hline Swath width & 30 km \\
\hline
\end{tabular}

Used SAR image is acquired in Interferometric Wide Swath Mode (IW) and GRD file type is selected. The properties of used SAR image are given in Table 2.

Table 2. Features of the S1A level-1 product GRD

\begin{tabular}{|l|l|}
\hline Date: & 04.11 .2016 \\
\hline Instrument: & SAR-C \\
\hline Operational mode: & IW swath mode \\
\hline Polarisation: & VH, VV \\
\hline Range and Azimuth Spacing: & $10 \mathrm{~m}$ \\
\hline Azimuth and Range Looks: & Single \\
\hline
\end{tabular}

Mosaic of the pansharpened RASAT image and SENTINEL-1A image of the study area are shown in Figure 2.
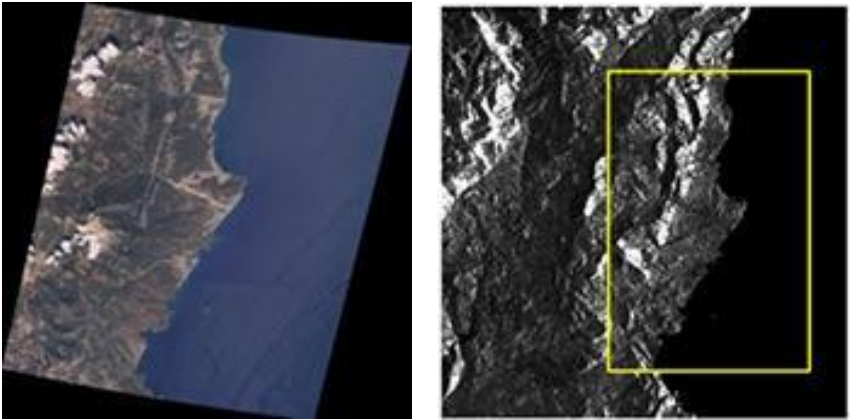

Figure 2. RASAT mosaic image (left), SENTINEL-1A image (right)

\subsection{Methodology Used}

Regarding the detection of shorelines from multispectral images, different image segmentation methods are proposed for different issues. In general, image segmentation methods can be classified into five groups which are (i) threshold based, (ii) edge based, (iii) region based, (iv) clustering techniques and (v) matching algorithms (Shih and Liu, 2016; Gong et al., 2015). Threshold method is the simplest one comparing to others. Many researchers developed different methods which are based on thresholding (Gao et al., 2016). Random Forest classifier is also an image segmentation method (Breiman, 2001) which is a data-driven method and enables efficient classifications using predictions produced from an ensemble of decision trees (Belgiu and Dragut, 2016). The Random Forests technique is based on the Bayesian tree or binary classification tree ( Cutler et al., 2007). In this study, the Random Forest classification has been realized by using Matlab ${ }^{\circledR}$ function TreeBagger. Random samples have been selected using bagging technique (bootstrap aggregation) for each node of the tree (Trigila et al., 2015). In this technique, $2 / 3$ of the training dataset is used as learning dataset (inBag) and $1 / 3$ is used for validation (Out-of-Bag (OOB)). In each node, the best branching is determined by using $\mathrm{m}$ random variables selected from all variables. This process uses the Calculation and Regression Tree (CART) algorithm. The CART algorithm uses the GINI index. For each node in a decision tree, the index determines the best interval and the impurity (He et al., 2015; Pal, 2005). The GINI index is defined according to (Pal, 2005) as in (1).

$$
\sum \sum(\mathrm{j} \neq \mathrm{i})(\mathrm{f}(\mathrm{Ci}, \mathrm{T}) /|\mathrm{T}|)(\mathrm{f}(\mathrm{Cj}, \mathrm{T}) /|\mathrm{T}|)
$$

In the equation, $\mathrm{T}$ is the learning data set, $\mathrm{Ci}$ is the class to which the pixel belongs, $\mathrm{f}(\mathrm{Ci}, \mathrm{T}) /|\mathrm{T}|$ is the probability that the selected pixel belongs to the class $\mathrm{Ci}$.

The number of trees and variables are chosen as 25 and 2, respectively. The RASAT image is classified with use of predict function derived from the decision tree. The training dataset contains 76605 in total since The RASAT image has 6744x7096 pixels.

The segmentation result from RASAT image is given in Figure 3. This result has been used as training data set to extract shoreline from the SENTINEL-1 image. 


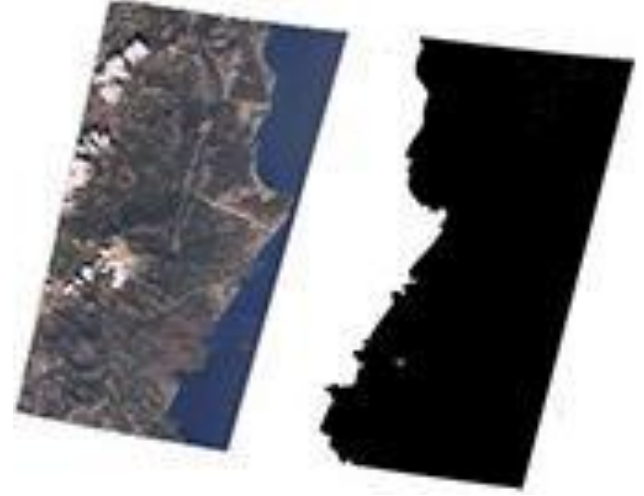

Figure 3. Land and Water-body segments

The proposed approach includes four processing steps: preprocessing, classification, post-processing and quality assessment. The flowchart of the method is shown in Figure 4. In the pre-processing step, cropping of the original image, speckle reduction, and geometric correction are performed. In the classification step, the fuzzy clustering using mean standard deviation method is applied to derive the shorelines with calculated parameters with use of RASAT based shorelines. In the post-processing step includes the generalization of the detected shorelines. The quality assessment covers the comparison of manually created shoreline with the final results.

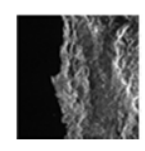

Input SAR image
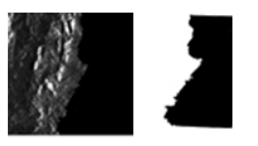

Classified Binary RASAT image

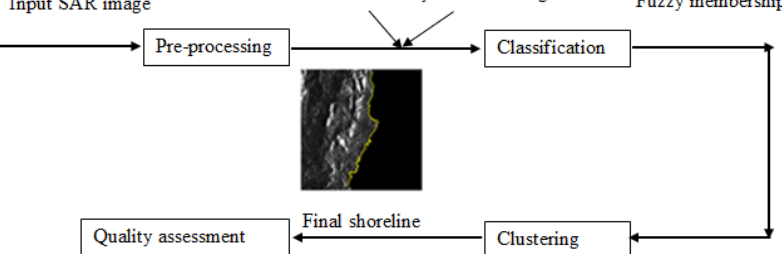

Figure 4. Flow chart of the shoreline detection from SAR image

The SAR images contain speckle which is needed to be reduced (Liu and Jezek, 2004). For this purpose, the Lee filter (Lee, 1986) is used since it is capable of reducing radar noise and speckle without losing the sharpness of the image. The image has been terrain-corrected with the use of SRTM terrain model (Figure 5).

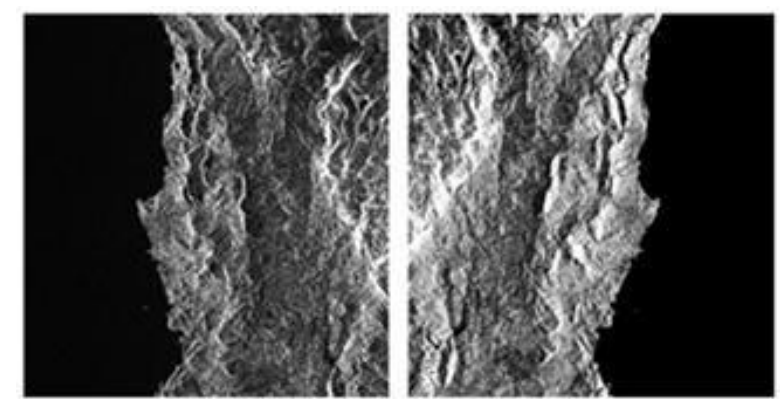

Figure 5. SAR imagery (left: original SAR data right: preprocessed SAR data

Shorelines are detected with the fuzzy approach. Fuzzy clustering classifies the images when converts the input data into a 0 to 1 scale, indicating the strength of a membership in a set, based on a designated type of fuzzy classification. MS Large function is used to calculate the membership value of the land surface class. This function is represented a fuzzy membership through a function based on the mean and standard deviation with the larger values having a membership closer to 1. Membership for the MS Large is computed the following equations;

$$
\begin{aligned}
& \begin{array}{l}
\text { if } \mathrm{x}>\mathrm{a} * \mathrm{~m}: \\
\mu(\mathrm{x})=1-(\mathrm{b} * \mathrm{~s}) /(\mathrm{x}-(\mathrm{a} * \mathrm{~m})+(\mathrm{b} * \mathrm{~s}))
\end{array} \\
& \begin{array}{l}
\mu(\mathrm{x})=0 \\
\text { where } \mathrm{m}=\text { the mean, }
\end{array} \\
& \mathrm{s}=\text { the standard deviation, a and } \mathrm{b} \text { multipliers denote input } \\
& \text { parameters. }
\end{aligned}
$$

Firstly, the mean and the standard deviation values are calculated for the whole SAR image. These parameters are 58 and 70 respectively. The result from RASAT image has been used as a mask, and a histogram of the SAR pixels of the masked region is shown in Figure 6.

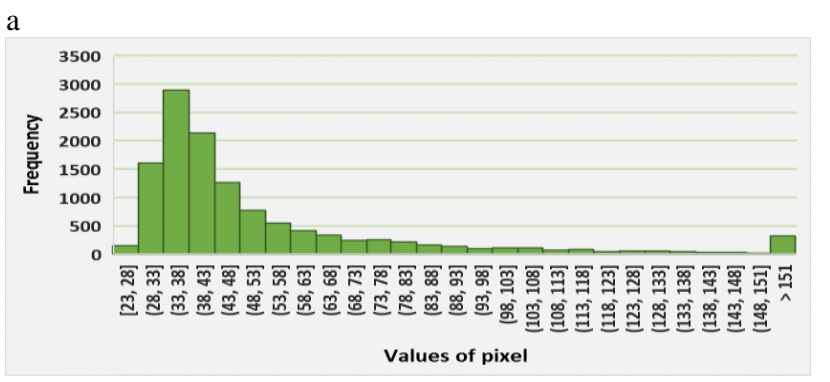

Figure 6. Histogram of water pixels from SAR data

Here, the histogram has peak values between 33 and 38. The mean of these values are selected as a parameter for fuzzy clustering, and multiplier parameters are defined as a: 0,60 and b: 0,003 to maximize the membership in Equation 2. Figure 7, shows the result of the clustering having two classes which are land and water. The centroid method is chosen to cluster the pixels as land pixels and the rest of water (Figure 8a). The boundary of the water class is generated as the shoreline. Then, the classified SAR image is converted raster to vector form.

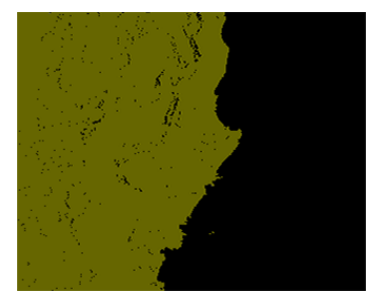

Figure 7. Extracted land and water clusters ( green: land surface, black: water surface)

After the conversion, the vector data include the zigzag effects which come from the raster data type and this effects is eliminated with Douglas-Peucker regularization algorithm (Dougles and Pecker, 1973)_(Figure 8) 


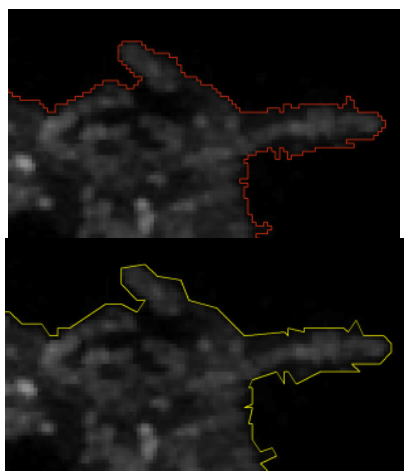

Figure 8. Elemination of zigzag effects (top:before, down:after)

\section{RESULTS}

The quality assessment has been performed with a comparison of the final result with the reference data which is manually created with use SENTINEL 1A data.

Details of the reference coastline and manually digitized coastline are presented in Figure 9. The extracted shorelines are converted to the dense points in every $10 \mathrm{~m}$. (which is spatial resolution of SAR image), and perpendicular distances to the reference shoreline are measured. Then statistics are calculated for the measured distances (Figure 10).
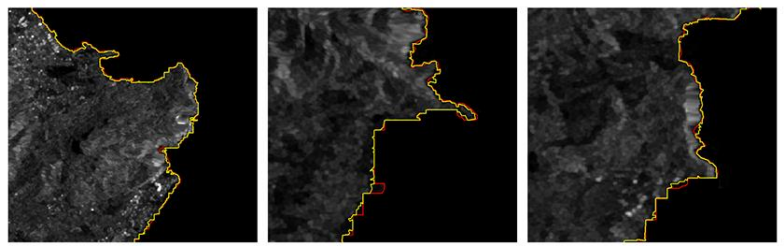

Figure 9. Extracted coastlines and manually digitized coastline details (yellow: SAR, red: manually created)
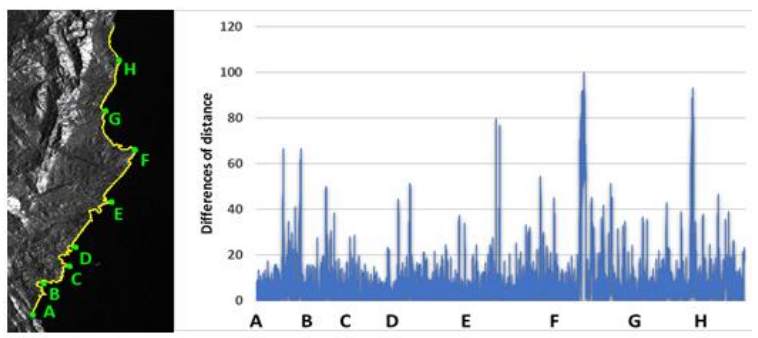

Figure 10. Differences of distance between the reference coastline and manually digitized coastline

The mean, standard deviation and median values between the final results and the reference vector data and between the initial results from RASAT and final result from SAR data are given in Table 3.

Table 3.Quality assessment of the results

\begin{tabular}{|l|c|c|}
\hline & $\begin{array}{c}\text { Reference Data - Result } \\
\text { from integrated approach } \\
(\mathrm{m})\end{array}$ & $\begin{array}{c}\text { Reference Data- } \\
\text { Result from } \\
\text { RASAT only } \\
(\mathrm{m})\end{array}$ \\
\hline Mean & 7.61 & 12.62 \\
\hline $\begin{array}{l}\text { Standard } \\
\text { Deviation }\end{array}$ & 11.01 & 13.39 \\
\hline Median & 4.28 & 8.55 \\
\hline
\end{tabular}

Obtained results have been compared with manually digitized data by calculating of perpendicular distances in $20 \mathrm{~cm}$ interval. According to results, the mean value of the distances between the final result and the reference data calculated as $5.59 \mathrm{~m}$, which is half pixel size of SENTINEL-1A. On the other hand, the mean of the distances between the reference and RASAT based shoreline results is calculated as $22.12 \mathrm{~m}$ which is three times larger than RASAT resolution.

Regarding the locations where there are large differences between the final result from SENTINEL-1A and the reference data, they are mainly caused by the terrain correction step. Because the SAR image has been terrain-corrected with the use of SRTM data which has lower resolution than the SAR image. So, the pixels which intersect with SRTM values on the locations where there are significant elevation changes moved from their original position much far away. Therefore, the shoreline result at these pixels has large differences with their correct position.

\section{CONCLUSIONS}

In this study, the medium resolution RASAT and SENTINEL imagery have been used to extract the shorelines with high precision. Extraction from SAR dataset itself would be difficult since the results are parameter sensitive and it is not easy to define the correct parameters for the precise results. On the other hand, extracting from RASAT has low accuracy compared to the final result from the combined use. Therefore, the combined use of RASAT and SAR images has significant capability to derive the shorelines with the quality of one pixel difference between reference dataset. RASAT image has been segmented by Random Forest method and results land/water body binary segmented image has been used for estimating the parameters used in fuzzy approach which is used to extract the shorelines from SENTINEL-1A image. Although the used RASAT pansharpened image has only visible bands, the performance of Random Forest classifier was very satisfying to create initial data for defining the fuzzy parameters for final segmentation step.

In the presented study, an integrated shoreline extraction approach has been proposed. Since extracted shorelines are used for detailed and temporal monitoring of coastal change, the proposed method can be used for coastal erosion studies, modelling of sediment transport and coastal morphodynamics.

The results are promising with good quality assessment results from the fuzzy approach. The determination of the parameters would be empirical in case the results from RASAT would not be used. SAR images are different than optical imagery to apply standard classification methods as performed in RASAT imagery. The images contain high level noise, so there is no identical information in the pixels regarding the objects. As shown in the quality assessment, fuzzy methods allow determining the different clusters with much separability between the classes.

In the future studies, other medium resolution image data (e.g. SENTINEL-2A) can be used to estimate the parameters of fuzzy membership function much precisely.

\section{ACKNOWLEDGEMENTS}

This study has been supported by TUBITAK (The Scientific and Technological Research Council of Turkey) with project number $115 \mathrm{Y} 718$. 


\section{REFERENCES}

Belgiu, M., \& Drăguț, L., 2016. Random forest in remote sensing: A review of applications and future directions. ISPRS Journal of Photogrammetry and Remote Sensing, 114, pp. 2431.

Breiman, L., 2001. Random forests. Machine learning, 45(1), pp. 5-32.

Erdogan, M., Yilmaz, A., Eker, O., 2016. The georeferencing of RASAT satellite imagery and some practical approaches to increase the georeferencing accuracy. Geocarto International, 31(6), pp. 647-660.

Cutler, D. R., Edwards, T. C., Beard, K. H., Cutler, A., Hess, K. T., Gibson, J., \& Lawler, J. J., 2007. Random forests for classification in ecology. Ecology, 88(11), pp. 2783-2792.

Douglas, D. Peucker, T., 1973. Algorithms for the reduction of the number of points required to represent a digitized line or its caricature, The Canadian Cartographer 10(2), 112-122.

Gao, H., Pun, C., Kwong, S., 2016. An efficient image segmentation method based on a hybrid particle swarm algorithm with learning strategy. Information Sciences, 369, pp. $500-521$.

Gong, M.G., Tian, D.Y., Su, L.Z., Jiao, L.C., 2015. An efficient bi-convex fuzzy variational image segmentation method. Information Sciences, 293, pp. 351-369.

He, J., Harris, J. R., Sawada, M., \& Behnia, P., 2015. A comparison of classification algorithms using Landsat-7 and Landsat-8 data for mapping lithology in Canada's Arctic. International Journal of Remote Sensing, 36(8), pp. 2252-2276.

Kim, D. J., Moon, W. M., Park, S. E., Kim, J. E., Lee, H. S., 2007. Dependence of waterline mapping on radar frequency used for SAR images in intertidal areas. IEEE Geoscience and Remote Sensing Letters, 4(2), pp. 269-273.

Lee, J. S., 1986. Speckle suppression and analysis for synthetic aperture radar images. Optical engineering, 25(5), pp. 255636255636.

Lee, J. S., \& Jurkevich, I., 1990. Coastline detection and tracing in SAR images. IEEE Transactions on Geoscience and Remote Sensing, 28(4), pp. 662-668.

Liu, H., \& Jezek, K. C., 2004. Automated extraction of coastline from satellite imagery by integrating Canny edge detection and locally adaptive thresholding methods. International Journal of Remote Sensing, 25(5), pp. 937-958.

Liu, Z., Li, F., Li, N., Wang, R., Zhang, H., 2016. A Novel Region-Merging Approach for Coastline Extraction From Sentinel-1A IW Mode SAR Imagery. IEEE Geoscience and Remote Sensing Letters, 13(3), pp. 324-328.

Mason, D. C., \& Davenport, I. J., 1996. Accurate and efficient determination of the shoreline in ERS-1 SAR images. IEEE Transactions on Geoscience and Remote Sensing, 34(5), pp. 1243-1253.

Modava, M., \& Akbarizadeh, G., 2017. Coastline extraction from SAR images using spatial fuzzy clustering and the active contour method. International Journal of Remote Sensing,
38(2), pp. 355-370.

Niedermeier, A., Romaneessen, E., Lehner, S., 2000. Detection of coastlines in SAR images using wavelet methods. IEEE Transactions on Geoscience and Remote Sensing, 38(5), pp. 2270-2281.

Paes, R. L., Nunziata, F., Migliaccio, M., 2015. Coastline extraction and coastal area classification via SAR hybridpolarimetry architecture. In: 2015 IEEE International Geoscience and Remote Sensing Symposium (IGARSS), Milan, Italy, pp. 3798-3801.

Pal, M., 2005. Random forest classifier for remote sensing classification. International Journal of Remote Sensing, 26(1), pp. 217-222.

Shu, Y., Li, J., Gomes, G., 2010. Shoreline extraction from RADARSAT-2 intensity imagery using a narrow band level set segmentation approach. Marine Geodesy, 33(2-3), pp. 187-203.

Shih, H.C., \& Liu, E.R., 2016. New quartile-based region merging algorithm for unsupervised image segmentation using color-alone feature. Information Sciences, 342, pp. 24-36.

Trigila, A., Iadanza, C., Esposito, C., \& Scarascia-Mugnozza, G., 2015. Comparison of Logistic Regression and Random Forests techniques for shallow landslide susceptibility assessment in Giampilieri. Geomorphology, 249, pp. 119-136.

Yu, Y., Acton, S. T., 2004. Automated delineation of coastline from polarimetric SAR imagery. International Journal of Remote Sensing, 25(17), pp. 3423-3438.

Xing, K., Y. Fu, \& F. Zhou., 2012. A New coastline extraction in remote sensing images. In: Bruzzone, L. (Ed.), Proceedings of Image and Signal Processing for Remote Sensing, Edinburgh, UK, Vol. XVIII, pp. 853718-9.

Zhang, H., Zhang, B., Guo, H., Lu, J., \& He, H., 2013. An automatic coastline extraction method based on active contour model. In: The 21st International Conference on Geoinformatics, Kaifeng, China, pp. 1-5. 